\title{
Behavioural activation delivered by the non- specialist: phase II randomised controlled trial
}

\author{
David Ekers, David Richards, Dean McMillan, J. Martin Bland and Simon Gilbody
}

\section{Background}

Behavioural activation appears as effective as cognitivebehaviour therapy (CBT) in the treatment of depression. If equally effective, then behavioural activation may be the preferred treatment option because it may be suitable for delivery by therapists with less training. This is the first randomised controlled trial to look at this possibility.

\section{Aims}

To examine whether generic mental health workers can deliver effective behavioural activation as a step-three highintensity intervention.

\section{Method}

A randomised controlled trial (ISRCTN27045243) comparing behavioural activation ( $n=24)$ with treatment as usual $(n=23)$ in primary care.

\section{Results}

Intention-to-treat analyses indicated a difference in favour of behavioural activation of $-15.79(95 \% \mathrm{Cl}-24.55$ to -7.02$)$ on the Beck Depression Inventory-II and Work and Social Adjustment Scale (mean difference $-11.12,95 \% \mathrm{Cl}-17.53$ to -4.70$)$.

\section{Conclusions}

Effective behavioural activation appears suitable for delivery by generic mental health professionals without previous experience as therapists. Large-scale trial comparisons with an active comparator (CBT) are needed.

\section{Declaration of interest}

None.
Depression will be the second largest cause of disease burden by $2020 .^{1}$ It affects between 5 and $10 \%$ of the population and is the third most common reason for primary care consultation. ${ }^{2}$ Psychological treatments, particularly cognitive-behavioural therapy (CBT), are recommended to treat depression; ${ }^{3,4}$ however, less than $10 \%$ of those affected receive such treatment. ${ }^{2}$ The standard CBT approach to depression is Beck's cognitive therapy, ${ }^{5}$ which uses both behavioural and cognitive strategies to identify, question and modify maladaptive thought processes, life rules and core beliefs. In a landmark study in 1996, Jacobson et al compared the full version of CBT with a reduced version that included some but not all cognitive components, and a third intervention, termed behavioural activation, that relied entirely on behavioural strategies. ${ }^{6}$ There was no evidence of any differences in effectiveness between treatments at post-treatment or follow-up. ${ }^{7}$ These findings led Jacobson et $a l^{6}$ and Jacobson \& Gortner $^{8}$ to put forward a parsimony argument in favour of behavioural activation: if CBT and behavioural interventions are equally effective, then behavioural ones may be preferable because they are simpler to deliver and can therefore be delivered more economically by professionals with less training. Were this to be the case, this would have substantial implications for the organisation and delivery of treatments. ${ }^{8}$ The provocative finding that much of what occurs in the leading psychological treatment for depression may be an unnecessary complication has led to a renewed interest in behavioural treatments. In a further study in 2006, Dimidjian and colleagues ${ }^{9}$ replicated the finding that CBT and behavioural activation were comparably effective, and a series of recent meta-analyses ${ }^{10-12}$ have also come to this conclusion. Although a main impetus for the renewed interest is the possibility of developing a more parsimonious treatment for depression, it is notable that no study reviewed in the meta-analyses has explored the parsimony argument in a controlled clinical trial. In earlier studies the treatments were delivered by clinicians who had previous experience of delivering therapy, with experience often amounting to several years. Although behavioural activation may indeed by simpler to deliver, it may be the experience of the therapist that counts. The aim of the current study was to examine whether generic mental health workers, without previous experience in therapy, could deliver effective behavioural interventions. We looked at the impact on depression symptom level, functioning and treatment satisfaction. This is, as far as we are aware, the first study to directly assess the parsimony argument offered in favour of behavioural activation.

\section{Method}

The Northumberland local research ethics committee and local NHS research governance departments approved this study. The trial is registered with the International Standard Randomised Controlled Trials Registry, number ISRCTN27045243 and complies with updated CONSORT recommendations. ${ }^{13}$ It was a 'phase II' randomised controlled trial of behavioural activation facilitated by generic mental health workers compared with usual care for adults with depression.

\section{Recruitment, participants and randomisation}

We recruited potential participants aged 18 or over from either general practices directly or from primary care mental health services over a 9-month period. Practices were based in a mix of rural and urban settings. These practices opted to participate in the trial after receiving information about it. Participants identified with depression and either on a stable dose or no dose of antidepressant medication for 6 weeks preceding identification were supplied with study information and if requested were referred into the trial by their general practitioner (GP) or mental health worker. Following consent, eligibility was confirmed by the use of a standardised computer-based assessment tool, the Clinical Interview Schedule Revised (CSIR), ${ }^{14}$ to confirm a ICD-10 15 diagnosis of depression. Exclusion criteria consisted of suicidal risk, psychotic symptoms, diagnosis of bipolar disorder, organic 
brain disease or the use of alcohol/non-prescription drugs requiring clinical intervention. Baseline measures were then taken.

Following assessment, participants were randomised to two arms through an allocation concealment process independent of the study team using a block randomisation system in blocks of four. Taking into account increased risk of a moderating effect of baseline severity of depression ${ }^{16}$ and because of the small sample sizes in this study, stratification based on baseline depression severity was conducted. Participants were allocated into two groups prior to randomisation based on Beck Depression Inventory (BDI-II $)^{17}$ scores $(\leqslant 25$ and $\geqslant 26)$. General practitioners and participants were informed of allocation automatically by letter.

\section{Sample size}

We calculated the sample size required based on those studies incorporating a delayed start to psychological interventions with variable levels of concurrent usual care rather than active placebo identified in our previous systematic review. ${ }^{11} \mathrm{~A}$ standardised between-group effect size (Cohen's $d$ ) of -0.84 (CI -1.27 to $-0.41)$ was observed in a sample of nine studies (282 participants) indicating that with alpha set at 0.05 to obtain $80 \%$ power, a sample size of 23 was required in each group.

\section{Measures}

The primary clinical outcome used for depression symptoms was the Beck Depression Inventory (BDI-II), ${ }^{17}$ with a score range of 0 to 63 (0-13 minimal, 14-19 mild, 20-28 moderate, 29-63 severe). Secondary outcome measures looked at functioning using the Work and Social Adjustment Scale (WSAS) ${ }^{18}$ and satisfaction using the Client Satisfaction Questionnaire (CSQ-8). ${ }^{19}$ Assessments were collected by a research worker masked to treatment allocation at baseline, 1-, 2- and 3-month follow-up. To reduce the risk of bias further we used self-completed assessments of depression symptom level, functioning and satisfaction.

\section{Interventions}

\section{Behavioural activation}

Participants received 12 1-hour face-to-face sessions of behavioural activation over a 3 -month period as a step-three intervention. ${ }^{3}$ Sessions followed a 12-session protocol based on two behavioural approaches developed in previous research..$^{2,21}$ Behavioural activation consisted of a structured programme increasing contact with potentially antidepressant environmental reinforcers through scheduling and reducing the frequency of negatively reinforced avoidant behaviours. A shared formulation was created based on a behavioural model in the early stages of treatment that was developed with the participant throughout the 12 sessions. Subsequent specific techniques incorporated in the 12-session protocol were self-monitoring, identifying 'depressed behaviours', developing alternative goal-orientated behaviours and scheduling. In addition, the role of avoidance and rumination was addressed through functional analysis and alternative responses were developed. The overall goal of behavioural activation was to re-engage participants with stable and diverse sources of positive reinforcement from their environment and to understand the behavioural activation rationale, thus developing depression management strategies for future use (the treatment manual is available from the author on request).

\section{Behavioural activation therapists}

Behavioural activation in this study was delivered by two qualified mental health nurses with no previous formal psychotherapeutic training or experience. Both had worked in a range of services in in-patient and community settings with 3 and 6 years experience since qualification. They received 5 days of training in behavioural activation and 1 hour of clinical supervision fortnightly from the principal investigator (D.E.). Training focused on the rationale and skills required to deliver a 12-session protocol of behavioural activation for depression. It included sections on behavioural learning theory and its application to depression, developing individualised behavioural activation formulations and specific techniques used in sessions. Training was a mix of presentation and role-play, with repeated practise and feedback. Competency assessment at the end of training was based on role-played treatment scenarios.

\section{Usual care}

Participants were followed up by their GP or primary care mental health worker and offered interventions deemed appropriate for their condition as per normal practice. At 3-month follow-up, control participants were offered behavioural activation therapy as delivered in the intervention arm.

\section{Adherence assessment}

All treatment sessions were audiotaped in the intervention arm. Recordings were stratified for study phase (early, mid, late) and therapists; $20 \%$ were then randomly selected by a research assistant masked to session content. Recordings of $38 \mathrm{~h}$ of therapy were then assessed by independent accredited cognitive-behavioural therapists with extensive experience in both CBT and behavioural activation. As no validated competency assessment tool is available for behavioural activation, we designed a brief checklist of treatment fidelity in this trial. Assessors specifically examined session and homework content against treatment protocols. They indicated if behavioural activation was the overall modality applied and if other therapeutic models were prominent in the therapy (such as cognitive therapy). We assigned scores of 1 where under each heading behavioural activation was dominant and scores of 0 if other therapy modes were prominent. After reviewing each tape, assessors decided if the session could be classed as behavioural activation with assigned values of 1 (yes) and (0) no.

\section{Data analysis}

Descriptive statistics are presented as means and standard deviations for psychometric scales (BDI-II, WSAS, CSQ-8) and counts and percentages for categorical variables (depressed/not depressed). The primary outcome variable, severity of depression at 3-month follow-up, was compared between groups using analysis of covariance on individual baseline depression (BDI-II) scores. Social functioning was compared at 3-month follow-up using analysis of covariance on individual baseline social adjustment scale scores. Satisfaction was measured at 3 months and compared between groups using an independent samples $t$-test. For continuous variables we present between-group mean end-point differences, both in terms of scores on the instrument and as standardised effect sizes (Cohen's $d$ ) and assigned values to effect size as per normal convention (small $0-0.32$, medium $0.33-0.55$, and large 0.56 and above). ${ }^{22}$ The data analysis approach was decided a priori using analysis of covariance to counter potential baseline variance that may influence results because of the small sample sizes in this study. 


\section{Missing data}

Missing data presents a common threat to the results of many trials, especially psychotherapy trials where sample sizes are small. Traditional approaches to dealing with missing data such as including completers only or last-observation-carried-forward (LOCF) can significantly bias results. ${ }^{23,24}$ To deal with such problems, we used, where possible, multiple imputation for our intention-to-treat analysis. ${ }^{25}$ We conducted an intention-to-treat analysis replacing missing data using multiple imputation by chained equations, as described by Royston using 100 imputations. $^{26}$ We incorporated baseline instrument scores (BDI-II, WSAS), age, gender, problem duration and allocation in modelling.

For the clinical significance analyses it was not possible to use the multiple imputations method as this approach does not supply individual participant-level data. We therefore report LOCF analyses for clinical significance as it is likely to be a conservative analysis.

\section{Clinical significance}

We used Jacobson \& $\operatorname{Truax}^{27}$ procedures for calculating reliable and clinically significance change to quantify clinical improvement in depressive symptoms on the BDI-II; this is recommended as a standard reporting strategy for all published research involving psychological interventions. ${ }^{28}$ This requires a pre- to post-treatment improvement in scores that is unlikely to be because of the inherent unreliability of the measure (reliable change) accompanied by a movement from a clinical range to a non-clinical one (clinically significant change). In calculating reliable and clinically significant change criteria, we used data from the BDI-II manual ${ }^{17}$ for clinical means, standard deviations and the reliability estimate (Cronbach's alpha), and data from Dozois et $a l^{29}$ for the nonclinical mean and standard deviation. On the basis of these data, a participant had to improve by ten points or more from pre- to post-treatment to show reliable change and in addition had to score 17 or above pre-treatment and 16 or below post-treatment to meet criteria for clinically significant change (see Jacobson \& $\operatorname{Truax}^{27}$ for details of calculations).
As an additional measure of clinical improvement we used the response and remission criteria given in Dimidjian et al. ${ }^{9}$ Response was defined as an improvement of at least $50 \%$ or more and remission as a score of $\leqslant 10$ on the BDI-II. Odds ratios with $95 \%$ confidence intervals were used to compare clinically significant change in the two groups.

\section{Results}

\section{Baseline characteristics}

In total, 68 participants were referred to the trial of whom 21 were excluded (17 did not meet diagnostic criteria, 2 refused randomisation, 2 had significant suicidal ideation (as measured by a score of $\geqslant 2$ on question 9 of the BDI-II)). Forty-seven participants met the inclusion criteria and proceeded to randomisation. Of these, 23 were allocated to behavioural activation and 24 to a control group. No differences were observed in scores at baseline between the two groups on BDI-II (mean for behavioural activation 35.57 (s.d. $=9.60)$ and for usual care 35.08 (s.d.=9.60)), WSAS (mean for behavioural activation 26.39 (s.d. $=7.30$ ) and for usual care 25.13 (s.d.=7.30)), CSIR (mean for behavioural activation 31 (s.d. =10.99) and for usual care 33.13 (s.d.=8.22)) or problem duration (mean for behavioural activation 186.91 weeks (s.d.=358.49) and for usual care 195.21 weeks (s.d.=404.64)). Baseline participant characteristics are presented in Table 1 and show that the participants in the trial are representive of individuals with long-term severe depression, with substantial impairment of functioning. Data were collected from 38 participants at 3-month assessment, 16 in the behavioural activation arm and 22 in the control arm. Of those opting out of the study, three did so post-randomisation (one behavioural activation group, two usual care group), three at 1 month (all in the behavioural activation group), and three at 2 months (all in the behavioural activation group). There were no significant differences between completers and those dropping out of treatment on baseline BDI-II depression scores (mean for those who dropped out 36.55 (s.d. $=10.77$ ) and for those who did not 35.21 (s.d. $=9.43)$ ) or duration of problem (mean for those who

\begin{tabular}{|c|c|c|c|}
\hline Baseline characteristic & $\begin{array}{l}\text { Behavioural activation } \\
\qquad(n=23)\end{array}$ & $\begin{array}{l}\text { Treatment as usual } \\
\qquad(n=24)\end{array}$ & $\begin{array}{c}\text { All } \\
(n=47)\end{array}$ \\
\hline Age, years: mean (range) & $46.43(24-63)$ & $43.08(28-63)$ & $44.72(24-63)$ \\
\hline \multicolumn{4}{|l|}{ Gender, $n(\%)$} \\
\hline Male & $8(35)$ & $10(41.7)$ & $18(38)$ \\
\hline Female & $15(65)$ & $14(58.3)$ & $29(62)$ \\
\hline \multicolumn{4}{|l|}{ Employment, $n$ (\%) } \\
\hline Full-time & $13(56.5)$ & $8(33.3)$ & $21(44.7)$ \\
\hline Part-time & $1(4.3)$ & $7(29.2)$ & $8(17)$ \\
\hline Housewife/husband & $1(4.3)$ & $1(4.2)$ & $2(4.3)$ \\
\hline Carer & 0 & $1(4.2)$ & $1(2.1)$ \\
\hline Retired & $3(13)$ & $3(12.5)$ & $6(12.8)$ \\
\hline Unemployed & $4(17.4)$ & $2(8.3)$ & $6(12.8)$ \\
\hline Incapacity benefit & $1(4.3)$ & $2(8.3)$ & $3(6.4)$ \\
\hline Duration of problem in weeks, mean (s.d.) & $186.91(358.49)$ & $195.21(404.64)$ & $191.15(378.61)$ \\
\hline Baseline Beck Depression Inventory-II score, mean (s.d.) & $35.57(9.60)$ & $35.08(9.60)$ & $35.32(9.50)$ \\
\hline Baseline Work and Social Adjustment Scale score, mean (s.d.) & $26.39(7.30)$ & $25.13(7.70)$ & $25.74(7.46)$ \\
\hline Baseline Clinical Interview Schedule Revised score, mean (s.d.) & $31(10.99)$ & $33.12(8.22)$ & $32.09(9.63)$ \\
\hline Prescribed antidepressants, $n(\%)$ & $15(65)$ & $17(71)$ & $32(68)$ \\
\hline \multicolumn{4}{|l|}{ Baseline Clinical Interview Schedule Revised (ICD-10) diagnosis, $n$ (\%) } \\
\hline Mild depression & $1(4.3)$ & $2(8.3)$ & $3(6.4)$ \\
\hline Moderate depression & $13(56.5)$ & $9(37.5)$ & $22(46.8)$ \\
\hline Severe depression & $8(34.8)$ & $13(54.2)$ & $21(44.7)$ \\
\hline Mixed anxiety and depression & 1 (4.3) & 0 & $1(2.1)$ \\
\hline
\end{tabular}


dropped out 182 weeks (s.d. $=439)$ and for those who did not 193 weeks $($ s.d. $=369))$. Of the 23 participants randomised to behavioural activation, 11 received all 12 sessions. Of those with missed sessions, three received one to three sessions, three received four to six sessions and five received seven to nine sessions. The study flow is presented in Fig. 1.

\section{Treatment integrity}

All reviewed sessions scored 1 (behavioural activation dominant) in relation to session and homework content and 0 in relation to other therapy modes being prominent; with all sessions scored 1 being classed as an example of behavioural activation.

\section{Additional interventions}

Antidepressant medication was prescribed at baseline to 17 (71\%) participants in the usual care group and $15(65 \%)$ participants in the behavioural activation group compared with 15/24 (62.5\%) and $12 / 23(52 \%)$ respectively during the intervention phase. Six participants in the usual care group had follow-up from a community psychiatric nurse. Two participants in the behavioural activation group had one session each with a psychiatrist.

\section{Depression symptom level post-treatment on the BDI-II}

A one-way between-groups analysis of covariance was conducted with participant's scores on the BDI-II pre-treatment used as the covariate (behavioural activation group $n=16$, usual care group $n=22$ ). After adjusting for baseline BDI-II scores there was a significant difference in favour of behavioural activation of -15.65 points on the BDI-II $(95 \%$ CI -6.90 to -24.41 , $P=0.001)$ representing a large effect size (Cohen's $d=-1.15$, $95 \%$ CI -0.45 to -1.85 ) (Table 2).

\section{Multiple imputation analysis of missing data on the BDI-II}

Intention-to-treat analyses with multiple imputation showed a mean difference on post-BDI-II scores of -15.78 in favour of behavioural activation (95\% CI -24.55 to $-7.02, P=0.001$ ), with all randomised participants (behavioural activation group $n=23$, usual care group $n=24$ ) included in the analysis.

\section{Clinically significant improvement on the BDI-II}

Using LOCF $(n=47), 65.2 \%$ of the behavioural activation group showed reliable improvement compared with $33.3 \%$ of the control group (odds ratio $(\mathrm{OR})=3.8,95 \%$ CI 1.1-12.5). Although more of the treatment group (43.5\%) met criteria for reliable and clinically significant change than the control group (20.8\%), the confidence interval for the odds ratio included $1(\mathrm{OR}=2.9,95 \%$ CI $0.8-10.6)$. Response rates were higher in the treatment group $(47.8 \%$ v. $16.7 \%, \mathrm{OR}=4.6,95 \%$ CI $1.2-17.7)$ and were on the border of significance for remission $(39.1 \% v .12 .5 \%, \mathrm{OR}=4.5$, $95 \%$ CI 1.0-19.6). Four participants (16\%) in the usual care group demonstrated deterioration at 3 months, which was not observed in the behavioural activation group. Figure 2 summarises pre- to post-treatment change against reliable and clinically significant criteria.

\section{Functioning post-treatment on the WSAS}

A one-way between-groups analysis of covariance was conducted with participant's scores on the WSAS pre-treatment used as the covariate (behavioural activation group $n=16$, usual care group $n=22$ ). After adjusting for baseline WSAS scores, there was a significant difference in favour of behavioural activation of -11.56 points $(95 \% \mathrm{CI}-4.79$ to $-18.33, P=0.001)$.

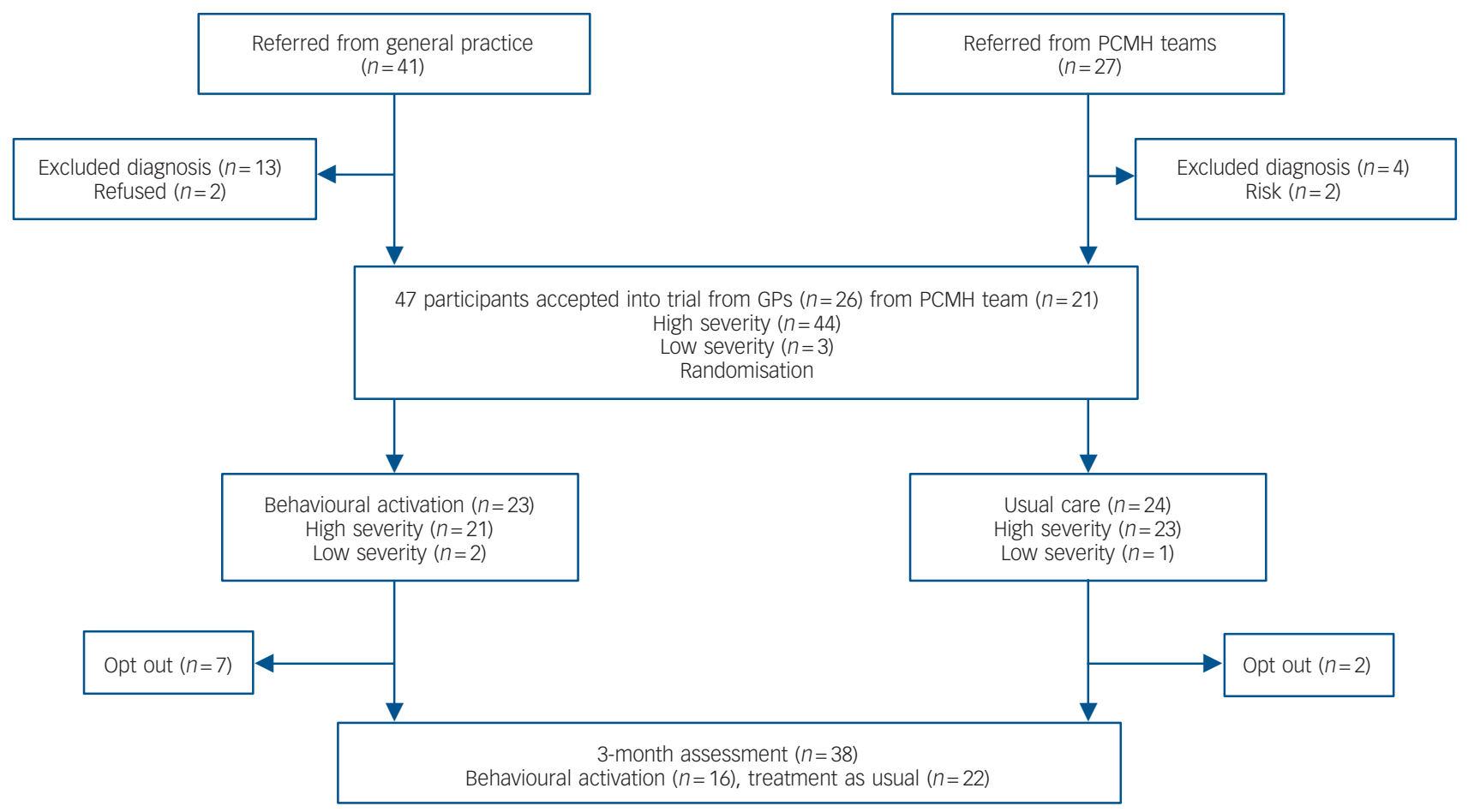

Fig. 1 Study flow chart. PCMH, primary care mental health; GPs, general practitioners. 

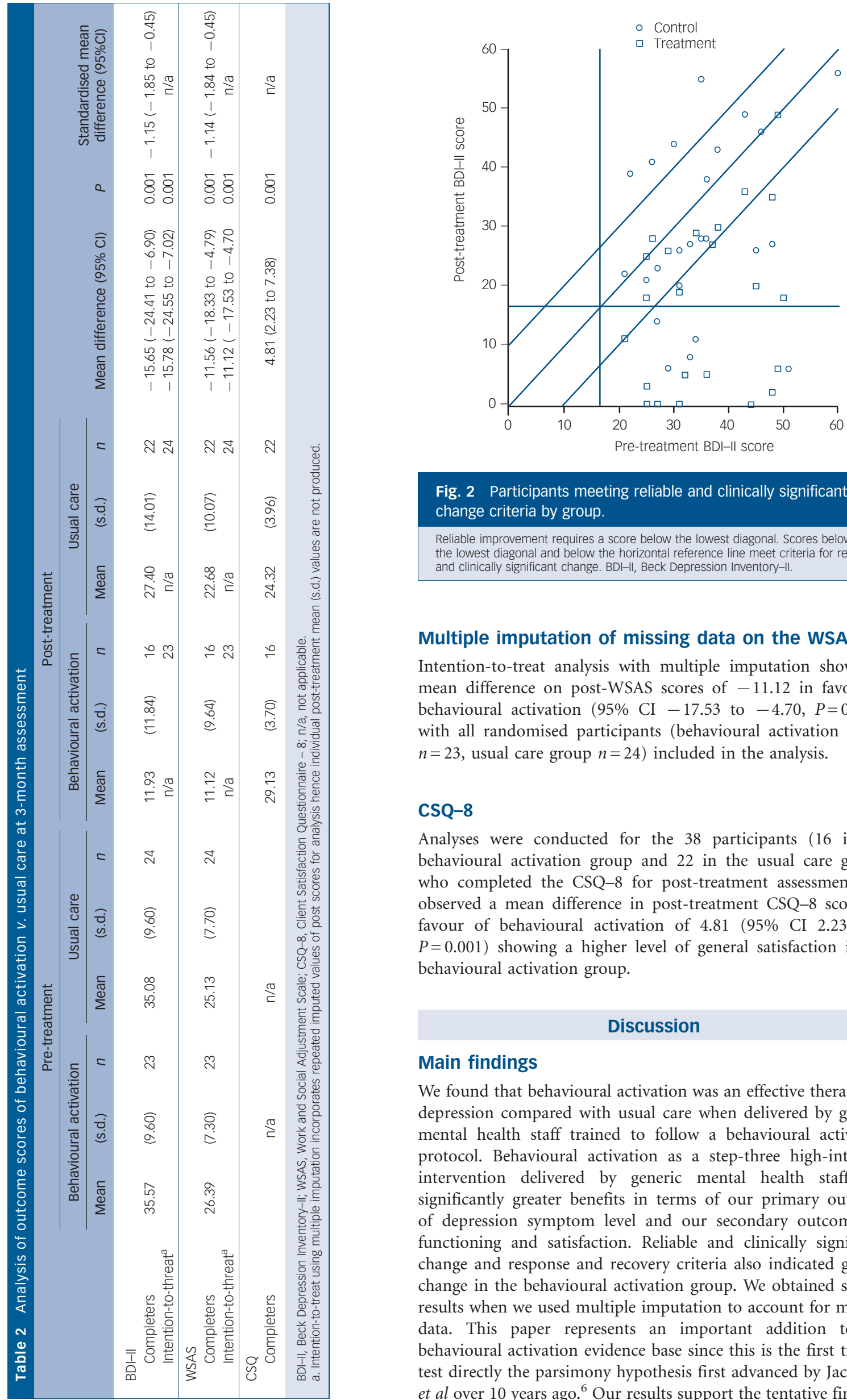

Fig. 2 Participants meeting reliable and clinically significant change criteria by group.

Reliable improvement requires a score below the lowest diagonal. Scores below the lowest diagonal and below the horizontal reference line meet criteria for reliable and clinically significant change. BDI-II, Beck Depression Inventory-II.

\section{Multiple imputation of missing data on the WSAS}

Intention-to-treat analysis with multiple imputation showed a mean difference on post-WSAS scores of -11.12 in favour of behavioural activation (95\% CI -17.53 to $-4.70, P=0.001$ ), with all randomised participants (behavioural activation group $n=23$, usual care group $n=24$ ) included in the analysis.

\section{CSQ-8}

Analyses were conducted for the 38 participants (16 in the behavioural activation group and 22 in the usual care group) who completed the CSQ-8 for post-treatment assessment. We observed a mean difference in post-treatment CSQ-8 scores in favour of behavioural activation of 4.81 (95\% CI 2.23-7.38, $P=0.001)$ showing a higher level of general satisfaction in the behavioural activation group.

\section{Discussion}

\section{Main findings}

We found that behavioural activation was an effective therapy for depression compared with usual care when delivered by generic mental health staff trained to follow a behavioural activation protocol. Behavioural activation as a step-three high-intensity intervention delivered by generic mental health staff had significantly greater benefits in terms of our primary outcome of depression symptom level and our secondary outcomes of functioning and satisfaction. Reliable and clinically significant change and response and recovery criteria also indicated greater change in the behavioural activation group. We obtained similar results when we used multiple imputation to account for missing data. This paper represents an important addition to the behavioural activation evidence base since this is the first trial to test directly the parsimony hypothesis first advanced by Jacobson et al over 10 years ago. ${ }^{6}$ Our results support the tentative findings 
of Boer et $a l^{30}$ that paraprofessionals may be able to deliver psychological therapies as effectively as professionals. Large, adequately powered trials are required to examine this further.

The various clinical significance criteria used in this study also provide support for behavioural activation delivered by generic workers in comparison with usual care. Differences between the groups were significant or close to significant for nearly all comparisons using both Jacobson \& Truax ${ }^{27}$ reliable and clinically significant change criteria and the response and remission criteria. In addition, we found an effect size of -1.15 on depression symptom level post-treatment favouring behavioural activation. This compares favourably with an overall effect size of -0.70 of 12 studies (459 participants) comparing behavioural activation with controls, using experienced therapists in our previously reported meta analysis. ${ }^{11}$

We also observed similar findings in terms of functioning; although both groups improved, in the behavioural activation group improvement was substantial, whereas in the usual care group this was marginal. There was a significant difference in favour of behavioural activation at 3 months, suggesting less functional impairment in this group.

Satisfaction with behavioural activation appeared extremely good with a mean of 29 on a 32-point scale, significantly better than usual care, although this also received a reasonably positive evaluation. This finding would suggest that alongside the clinical gains achieved by behavioural activation delivered by generic mental health workers, those receiving the intervention found the experience very acceptable.

Participants in the study had high baseline depression of long duration representing a highly complex clinical group with longerterm and recurrent depression. Therapists were representative of the vast pool of generic mental health workers in that they had no previous therapy training, were relatively recently qualified and employed at the base level of registered psychiatric nurses. They delivered behavioural activation to a high standard in accordance with a 12-session protocol. Based on independent evaluation it would appear that with very little training it is possible to equip staff with the skills to deliver behavioural activation that is both acceptable and clinically effective.

\section{Study limitations}

This was an exploratory study and there are a number of limitations of note. First, the relatively small numbers of participants and therapists recruited limits the generalisability of results. We did however base numbers on power calculations from our previous meta-analysis suggesting a sample size of 23 in each arm was sufficient to detect previously observed effect sizes. Despite this, it would have been beneficial to have recruited more participants to allow for our completers analysis to reflect these numbers rather than our intention-to-treat analysis. The small sample size may account for the wide $95 \%$ confidence intervals found in this study post-treatment. These should be considered when reflecting on findings; future larger trials should provide more precise estimates of difference.

We found that more people dropped out in the behavioural activation group than in the usual care group. This in itself is not surprising as behavioural activation is an active intervention relying on the person receiving treatment to complete homework on a regular basis. Of those discontinuing treatment, three did so in the first month and three in the second. The drop-out rate in this study is similar to those seen in large data-sets of CBT provision in primary care. ${ }^{31}$ Usual care in contrast does not involve as much investment from the participant and is nested within a person's overall healthcare, hence reducing the likelihood of drop out. Larger sample sizes however would have allowed us to have a more precise estimate of this finding as it is likely our limited sample was underpowered to accurately estimate likely drop-out from behavioural activation delivered by this workforce.

We used a self-report measure as our analysis of depression symptom level post-intervention. This is a source of potential information bias as in psychological therapy trials participants will be aware of their treatment allocation; this should be balanced in future studies by a repeated diagnostic interview. The lack of follow-up is also a limiting factor in the interpretation of the study. Previous studies of behavioural activation have demonstrated its durability to be equal to other therapies such as CBT; however, such studies have been delivered by experienced therapists. The aim of this study was to explore feasibility and parsimony of behavioural activation, although we found results supporting this we were unable to conduct follow-up assessments, which must be accounted for in future research. We were also unable to incorporate any validated measure of behavioural activation competence. This would have been beneficial to further asses the quality of behavioural activation administered and any possible contamination of the treatment modality.

\section{Clinical implications and future research}

This study is the first randomised controlled clinical trial to test feasibility of dissemination of behavioural activation to a wider mental health workforce and as such represents a major step forward in our understanding of this intervention. We have demonstrated that with limited training, generic mental health workers can be trained to deliver clinically effective behavioural activation to people with severe long-standing depression. If these findings can be replicated and translated into routine healthcare, then the clinical and cost implications of this finding are substantial for this prevalent and disabling condition. Now that such feasibility has been shown, future research with a larger sample and multiple therapists should investigate its longer-term durability and compare this behavioural activation delivery mode with an active psychological treatment such as CBT.

David Ekers, MSc, Dip CBT, RMN, Tees Esk and Wear Valleys NHS Trust and Menta Health Research Centre Durham University, $C$ Durham; David Richards, PhD, RN, School of Psychology, University of Exeter, Exeter; Dean McMillan, DclinPsy, PhD, Department of Health Sciences, Hull York Medical School and Department of Health Sciences, University of York, York; J. Martin Bland, MSC PhD, Department of Health Sciences, University of York, York; Simon Gilbody, DPhil, FRCPsych, Psychological Medicine and Health Services Research, Hull York Medical School and Department of Health Sciences, University of York, York, UK

Correspondence: David Ekers, Mental Health Research Centre, Durham University, Health Centre, Chester Le Street, Co Durham, DH3 3UR, UK. Email: david.ekers@tewv.nhs.uk

First received 23 Feb 2010, final revision 2 Aug 2010, accepted 11 Aug 2010

\section{References}

1 World Health Organization. The World Health Report 2001: Mental Health: New Understanding, New Hope. WHO, 2001

2 Singleton N, Bumpstead R, O'Brien M, Lee A, Meltzer Y. Office of National Statistics: Psychiatric Morbidity Among Adults Living in Private Households. HMSO, 2001.

3 National Institute for Health and Clinical Excellence. Management of Depression in Primary and Secondary Care. NICE, 2009.

4 Hollon S, Thase M, Markowitz J. Treatment and prevention of depression. Psychol Sci Public Interest 2002; 3: 1-39.

5 Beck A. Cognitive Therapy and the Emotional Disorders. International Universities Press, 1976. 
6 Jacobson N, Dobson K, Truax P, Addis M, Koerner K, Gollan J, et al. A component analysis of cognitive-behavioral treatment for depression. J Consult Clin Psychol 1996; 64: 295-304.

7 Gortner E, Gollan J, Dobson K, Jacobson N. Cognitive-behavioral treatment for depression: relapse prevention. J Consult Clin Psychol 1998; 66: 377-84.

8 Jacobson N, Gortner E. Can depression be de-medicalised in the 21st century: scientific revolutions, counter revolutions and the magnetic field of normal science. Behav Res Ther 2000; 38: 103-17.

9 Dimidjian S, Hollon S, Dobson K, Schmaling K, Kohlenberg R, Addis M, et al. Randomized trial of behavioural activation, cognitive therapy, and antidepressant medication in the acute treatment of adults with major depression. J Consult Clin Psychol 2006; 74: 658-70.

10 Cuijpers $P$, van Strate A, Warmerdam L. Behavioural activation treatments of depression: a meta analysis. Clin Psychol Rev 2007; 27: 318-26.

11 Ekers D, Richards D, Gilbody S. A meta analysis of behavioural therapy for depression. Psychol Med 2008; 38: 611-23.

12 Mazzucchelli T, Kane R, Rees C. Behavioral activation treatments for depression in adults: a meta-analysis and review. Clin Psychol Sci Pract 2009; 16: 383-411.

13 Schulz K, Altman D, Moher D. CONSORT 2010 Statement: updated guidelines for reporting parallel group randomised trials. BMJ 2010; 340: 698-702.

14 Lewis G, Pelosi A, Araya R, Dunn G. Measuring psychiatric disorder in the community: a standardized assessment tool for use by lay interviewers. Psychol Med 1992; 22: 465-86.

15 World Health Organization. The ICD-10 Classification of Mental and Behavioural Disorders: Clinical Descriptions and Diagnostic Guidelines. WHO 1992.

16 Elkin I, Gibbons R, Shea M, Sotsky S, Watkins J, Pilkonis P, et al. Initial severity and differential treatment outcome in the National Institute of Mental Health Treatment of Depression Collaborative Research Program. J Consult Clin Psychol 1995; 63: 841-7.

17 Beck A, Steer R, Brown G. Manual for Beck Depression Inventory II (BDI-II). Psychology Corporation, 1996.

18 Mundt JC, Marks IM, Shear MK, Greist JM. The Work and Social Adjustment Scale: a simple measure of impairment in functioning. Br J Psychiatry 2002; 180: 461-4.
19 Attkisson C, Greenfield T. The UCSF Client Satisfaction Scales: I. The Client Satisfaction Questionnaire-8. In The Use of Psychological Testing for Treatment Planning and Outcome Assessment, 3rd edn (ed M Maruish): 799-812. Lawrence Erlbaum Associates, 2004

20 Martell C, Addis M, Jacobson N. Depression in Context. Strategies for Guided Action. Norton, 2001.

21 Hopko D, Lejuez C, Ruggiaro K, Eifert G. Contemporary behavioural activation treatments for depression: procedures, principles and progress. Clin Psychol Rev 2003; 23: 699-717.

22 Lipsey M, Wilson D. The efficacy of psychological, educational and behavioural treatment. Am Psychol 1993; 48: 1181-209.

23 Nüesch E, Trelle S, Richenbach S, Rutjes A, Burgi E, Scherer M, et al. The effects of excluding patients from the analysis in randomised controlled trials: meta-epidemiological study. BMJ 2009; 339: b3244.

24 Streiner $D$, Geddes J. Intention to treat analysis in clinical trials when there are missing data. Evid Based Ment Health 2001; 4: 70-1.

25 Sterne J, White I, Carlin J, Spratt M, Royston P, Kenwad M, et al. Multiple imputation for missing data in epidemiological and clinical research: potential and pitfalls. BMJ 2009; 339: b2393.

26 Royston P. Multiple imputation of missing values. Stata J 2004; 4: 227-41.

27 Jacobson N, Truax P. Clinical significance: a statistical approach to defining meaningful change in psychotherapy research. J Consult Clin Psychol 1991; 59: 12-9.

28 Evans $\mathrm{C}$, Margison $\mathrm{F}$, Barkham $\mathrm{M}$. The contribution of reliable and clinically significant change methods to evidence-based mental health. Evid Based Ment Health 1998; 1: 70-2.

29 Dozois J, Dobson K, Ahnberg JA. A psychometric evaluation of the Beck Depression Inventory-II. Psychol Assess 1998; 10: 83-9.

30 Boer P, Wiersma D, Russo S, Bosch R. Paraprofessionals for anxiety and depressive disorders. Cochrane Database Syst Rev 2005; 2: CD004688.

31 Clark D, Layard R, Smithies R, Richards D, Suckling R, Wright B. Improving access to psychological therapy: Initial evaluation of two UK demonstration sites. Behav Res Ther 2009; 47: 910-20.

\section{Alexandra Pitman}

Edvard Munch is best known for The Scream, 1893, an image endlessly reproduced in the media to depict mental anguish. Explanations of the meaning behind the image abound, mainly focusing on an outpouring of emotion in response to suffering. Munch's own explanation is revealed in his diaries, which recall the melancholy of a walk along a bridge with friends. Trembling in fear at the fiery sunset, he sensed 'how an infinite scream was going through the whole of nature'. This dehumanised figure, into which viewers project their own neuroses, is not screaming but blocking out the scream of its existence. 